\title{
Formation of dynamical structures in relativistic jets: the FRI case
}

\author{
P. Rossi ${ }^{1}$, A. Mignone ${ }^{1,2}$, G. Bodo ${ }^{1}$, S. Massaglia ${ }^{2}$, and A. Ferrari ${ }^{2,3}$ \\ 1 INAF/Osservatorio Astronomico di Torino, Strada Osservatorio 20, 10025 Pino Torinese, Italy \\ e-mail: rossi@oato.inaf.it \\ 2 Dipartimento di Fisica Generale, Università degli Studi di Torino, via P. Giuria 1, 10125 Torino, Italy \\ 3 Department of Astronomy and Astrophysics, University of Chicago, 5640 S. Ellis, Chicago, IL 60637, USA
}

Received 29 February 2008 / Accepted 30 May 2008

\begin{abstract}
Context. Strong observational evidence indicates that all extragalactic jets associated with AGNs move at relativistic speed up to $100 \mathrm{pc}^{-1} \mathrm{kpc}$ scales from the nucleus. At greater distances, reflecting the Fanaroff-Riley radio source classification, we observe an abrupt deceleration in FR-I jets while relativistic motions persist up to Mpc scale in FR-II. Moreover, VLBI observations of some object such as B2 1144+35, Mrk501 and M87 show limb brightening of the jet radio emission on the parsec scale. This effect is interpreted kinematically as due to the deboosted central spine at high Lorentz factor and of a weakly relativistic external layer. Aims. In this paper we investigate whether these effects can be interpreted by a braking of the collimated flow by external medium entrainment favored by shear instabilities, namely Kelvin-Helmholtz instabilities. We examine in details the physical conditions under which significant deceleration of a relativistic flow is produced.

Methods. We investigated the phenomenon by means of high-resolution three-dimensional relativistic hydrodynamic simulations using the PLUTO code for computational astrophysics.

Results. We find that the most important parameter in determining the instability evolution and the entrainment properties is the ambient/jet density contrast. We show that lighter jets suffer stronger slowing down in the external layer than in the central part and conserve a central spine with a high Lorentz factor.

Conclusions. Our model is verified by constructing synthetic emission maps from the numerical simulations which compare reasonably well with VLBI observations of the inner part of FR-I sources.
\end{abstract}

Key words. galaxies: jets - hydrodynamics - instabilities - methods: numerical

\section{Introduction}

Extragalactic radio sources are traditionally divided into two morphological classes according to their intrinsic power (Fanaroff \& Riley 1974): Low luminosity sources (Fanaroff-Riley type I, FR-I) are brighter close to the nucleus of the parent galaxy and their jets become dimmer with distance, while high power sources (Fanaroff-Riley type II, FR-II) show the maximum brightness in the hot spots at the jet termination. The different morphology is generally accepted as reflecting a difference in how the jet energy is dissipated during propagation in the extragalactic medium, and it produces the observed radiation. For FR-I sources, it was quickly accepted that entrainment and deceleration of the jet must play an important role in shaping their morphology (Bicknell 1984, 1986; De Young 1996; Komissarov 1994), while in FR-II sources energy and momentum are transported without losses to the front working surface.

More recently a large body of evidence has accumulated showing that jets are relativistic at their base, not only in FR-II radio sources but also in FR-I. Superluminal motions are observed on milliarcsecond scales in several FR-I jets (Giovannini et al. 2001) and on arcsecond scales in M87 and Cen A (Biretta et al. 1995; Hardcastle et al. 2003); finally on small scales, there are also observations of one-sidedness and brightness asymmetry between jet and counter-jet, most likely due to Doppler boosting effects (Laing et al. 1999). The FR-I sources are also thought to be the parent population of BL Lac objects, for which the presence of relativistic velocities on parsec scales is well established (Urry \& Padovani 1995). The Lorentz factors of the jet bulk motion at sub-pc scales cannot be deduced directly from the observations, but are inferred from assumption of physical emission mechanism. In their review, Harris \& Krawczynski (2006) indicate that FR-II radiogalaxies have jets with bulk Lorentz factors between 5 and 40 (see also Urry \& Padovani 1995) and that FR-I jets have less constrained values that are somewhat lower than those of the FR-II jets. With data on proper motions and brightness ratio between jet and counter jet in a sample of radiogalaxies, Giovannini et al. (2001) do not find any systematic difference between low and high power radio sources, and the values they derive are between 3 and 10 . Celotti \& Ghisellini (2008) derive jet physical properties modeling the spectral energy distribution in a sample of blazars and find no difference between BL Lac's (associated to low power radio sources in the unified model) and radio-quasars (associated to high power radio sources), with an average value of about 15 .

On the other hand, relativistic motions from the inner regions all the way to larger scales, with Lorentz factors of about ten, appear to be present in powerful FR-II jets, as indicated by Chandra's discovery of bright X-ray emission on kpc scales (Tavecchio et al. 2004; Harris \& Krawczynski 2006); instead sub-relativistic velocities are found on kpc scales (Bicknell 1994) in low power radio sources, and the decrease in brightness asymmetry along the jet suggests that a deceleration must occur (Laing et al. 1999; Laing \& Bridle 2002; Canvin et al. 2005).

Both these morphological and kinematical data are indicative of the interaction between collimated outflows and the 
surrounding medium. In particular jet deceleration can be obtained by redistributing the bulk momentum through some form of mass entrainment (Bicknell 1994, 1995). Velocity shear instabilities are the most likely triggering mechanisms of entrainment (De Young 1996, 2005) $)^{1}$. In fact the nonlinear development of velocity shear or Kelvin-Helmholtz instabilities leads to an exchange of mass, momentum and energy at contact discontinuities between fluids in relative motion (Bodo et al. 1994, 1995, 1998); understanding the details of this process is essential for modeling the jet dynamics and for giving clues on the determination of the jet physical parameters. One of the key features of this form of interaction is the formation of a mixing layer at the interface between jet and surrounding medium, where the external material is entrained and accelerated at the expense of the jet momentum. This process leads to the formation of a transverse velocity profile where the internal layers feel the effects of the interaction with the ambient medium less and keep an higher velocity, while the external layers are more decelerated. The presence of this transverse velocity structure has already been suggested for explaining some of the observational properties of radio sources such as their magnetic field configuration (Komissarov 1990; Laing 1993) and limb brightening effects (Giroletti et al. 2004). And it overcomes problems in unifying radiogalaxies with BL Lac objects (Chiaberge et al. 2000).

For studying the instability evolution and the subsequent entrainment process in detail one has to resort to three-dimensional numerical simulations, since the mechanisms at the base of the entrainment are inherently three-dimensional, as shown in preliminary analyses by Bodo et al. (2003) and Rossi et al. (2004).

In this paper we present results of high-resolution hydrodynamic simulations in which we follow the evolution of a perturbed relativistic jet as it propagates in a homogeneus stationary ambient medium. We assume that the jet is in pressure equilibrium with the outside medium, although this choice is not critical for the final results. Perturbations grow as a consequence of the velocity shear instabilities and lead to entrainment of the external medium and to jet deceleration. The main questions we address are the dependence of the entrainment and deceleration processes on the jet's physical parameters and the kind of structure that the jet acquires as the result of these processes.

The plan of the paper is the following: in Sect. 2 we present the numerical setup adopted for calculations and the parameter space covered by the simulations; in Sect. 3 we discuss the results of our simulations focusing our attention on the dependence of the efficiency of deceleration on the physical parameters; in Sect. 4 we analyze the entrainment properties in more detail for the case that appears more successful in decelerating the jet. Preliminary comparisons of our simulations with FR-I sources of different morphologies are reported in Sect. 5 and conclusions of our study are drawn in Sect. 6.

\section{Numerical setup}

Numerical simulations are carried out by solving the equations of particle number and energy-momentum conservation. Referring to the observer's reference frame, where the fluid moves with velocity $v_{k}$ (in units of the speed of light $c$ ) with

\footnotetext{
1 An alternative and more likely complementary view of the origin of the entrainment has been presented by Komissarov (1994) and Bowman et al. (1996), who consider the possibility of entrainment by the injection of mass lost by stars within the jet volume.
}

respect to the coordinate axis $k=x, y, z$ and assuming a flat metric, the conservation laws take the differential form:

$$
\frac{\partial}{\partial t}\left(\begin{array}{c}
\rho \gamma \\
w \gamma^{2} v_{k} \\
w \gamma^{2}-p \\
\rho \gamma f
\end{array}\right)+\sum_{i} \frac{\partial}{\partial x_{i}}\left(\begin{array}{c}
\rho \gamma v_{i} \\
w \gamma^{2} v_{k} v_{i}+p \delta_{k i} \\
w \gamma^{2} v_{i} \\
\rho \gamma f v_{i}
\end{array}\right)=0
$$

where $\rho, w, p$ and $\gamma$ denote, respectively, the rest mass density, enthalpy, gas pressure and Lorentz factor. The jet and external material are distinguished using a passive tracer, $f$, set equal to unity for the injected jet material and equal to zero for the ambient medium. The system of Eqs. (1) is completed by specifying an equation of state relating $w, \rho$ and $p$. Following Mignone et al. (2005), we adopt the following prescription:

$w=\frac{5}{2} p+\sqrt{\frac{9}{4} p^{2}+\rho^{2}}$

which closely reproduces the thermodynamics of the Synge gas for a single-specie relativistic perfect fluid offering, at the same time, considerable ease of implementation and reduced numerical cost (Mignone \& McKinney 2007).

Simulations were carried out on a Cartesian domain with coordinates in the range $x \in\left[-L_{x} / 2, L_{x} / 2\right], y \in\left[0, L_{y}\right]$ and $z \in\left[-L_{z} / 2, L_{z} / 2\right]$ (lengths are expressed in units of the jet radius, $y$ is the direction of jet propagation). At $t=0$, the domain is filled with a perfect fluid of uniform density and pressure values representing the external ambient medium initially at rest. A constant cylindrical inflow is initialized in the small region $y \leq 1, \sqrt{x^{2}+z^{2}} \leq 1$ with velocity along the $y$ coordinate and is constantly fed into the domain through the lower $y$ boundary. Jet inflows are represented by a three-parameter family distinguished by the beam Lorentz factor $\gamma_{\mathrm{b}}$, Mach number $M=v_{\mathrm{b}} / c_{\mathrm{s}}$ and the ambient-to-beam density contrast $\eta$. Thus, by normalizing the beam density to $\rho_{\mathrm{b}} \gamma_{\mathrm{b}}=1$, the ambient medium is given by $\rho_{\mathrm{a}}=\eta / \gamma_{\mathrm{b}}$. The beam is initially pressure-matched and $p_{\mathrm{b}}$ is recovered from the definition of the Mach number. This assumption is the most used in jet simulations and so we maintain it in this first study; however, it can be questioned, since it is not clear what the mechanism could be that brings pressure equilibrium between the jet and the surrounding medium. An alternative approach is suggested by Komissarov \& Falle (1998) and could be worth investigating in future studies. In our simulations we fixed the jet Lorentz factor equal to 10 at the jet inlet, a representative value in the range derived from the observations discussed in the Introduction. For the Mach number we choose the values of 3 and 30; however for relativistic fluids it is actually more appropriate to consider the relativistic Mach number (Konigl 1980; Komissarov \& Falle 1998) defined as $M_{\mathrm{r}}=\gamma_{\mathrm{b}} v_{\mathrm{b}} / \gamma_{\mathrm{s}} c_{\mathrm{s}}$, where $\gamma_{\mathrm{s}}=1 / \sqrt{1-c_{\mathrm{s}}^{2} / c_{2}}$. It is in fact $M_{\mathrm{r}}$ that has the same physical interpretation as the usual non-relativistic Mach number since it reflects the propagation of sound waves and the value of Mach angle. Finally for the density ratio we choose the values $10^{2}$ and $10^{4}$. The complete set of parameters for our simulations is given in Table 1.

At the jet inlet, transverse velocities are perturbed introducing pinching, helical and fluting modes with corresponding azimuthal $\left(\phi=\tan ^{-1}(z / x)\right)$ wave numbers $m=0,1,2$ :

$\left(v_{x}, v_{z}\right)=\frac{A}{24} \sum_{m=0}^{2} \sum_{l=1}^{8} \cos \left(m \phi+\omega_{l} t+b_{l}\right)(\cos \phi, \sin \phi)$, 
Table 1. Parameter set used in the numerical simulation model.

\begin{tabular}{crrrrccc}
\hline \hline Case & $\gamma_{\mathrm{b}}$ & $M$ & $M_{\mathrm{r}}$ & $\eta$ & pts/beam & $L_{x} \times L_{y} \times L_{z}$ & $N_{x} \times N_{y} \times N_{z}$ \\
\hline $\mathrm{A}$ & 10 & 3 & 28.3 & $10^{2}$ & 20 & $50 \times 150 \times 50$ & $324 \times 1200 \times 324$ \\
$\mathrm{~B}$ & 10 & 3 & 28.3 & $10^{4}$ & 20 & $60 \times 75 \times 60$ & $344 \times 600 \times 344$ \\
$\mathrm{C}$ & 10 & 3 & 28.3 & $10^{4}$ & 12 & $50 \times 75 \times 50$ & $172 \times 300 \times 172$ \\
$\mathrm{D}$ & 10 & 30 & 300 & $10^{4}$ & 20 & $50 \times 150 \times 50$ & $324 \times 1050 \times 324$ \\
$\mathrm{E}$ & 10 & 30 & 300 & $10^{2}$ & 12 & $24 \times 200 \times 24$ & $144 \times 560 \times 144$ \\
\hline
\end{tabular}

where high $(l=1, \ldots, 4)$ and low $(l=5, \ldots, 8)$ frequency modes are given, respectively, by $\omega_{l}=c_{\mathrm{s}}(1 / 2,1,2,3)$ and $\omega_{l}=c_{\mathrm{s}}(0.03,0.06,0.12,0.25)$. The phase shifts $b_{l}$ are randomly chosen. The amplitude $A$ of the perturbation corresponds to a fractional change in the bulk Lorentz factor, $\gamma_{\mathrm{b}} \rightarrow \gamma_{\mathrm{b}}(1+\epsilon)$, yielding

$A=\frac{\sqrt{(1+\epsilon)^{2}-1}}{\gamma_{\mathrm{b}}(1+\epsilon)}$.

We typically set $\epsilon=0.05$. Outside the inlet region we impose symmetric boundary conditions (emulating the presence of a counter-jet), whereas the flow can freely leave the domain throughout the remaining boundaries.

Explicit numerical integration of the equation system (1) is achieved using the relativistic hydrodynamics module available in the PLUTO code (Mignone et al. 2007). PLUTO is a Godunov-type, multi-physics code providing a variety of computational algorithms for the numerical integration of hyperbolic conservation laws in multiple spatial dimensions. For the present application, we employ the dimensionally split version of the relativistic Piecewise Parabolic Method (PPM) presented in Mignone et al. (2005), which has an overall 2nd order accuracy in both space and time.

The physical domain is covered by $N_{x} \times N_{y} \times N_{z}$ computational zones that are not necessarily uniformly spaced. For domains with a large physical size, we employ a uniform grid resolution around the beam (typically for $|x|,|z| \leq 2$ ) and a geometrically stretched grid elsewhere.

\section{Results}

The overall characteristics of relativistic jet propagation have been originally discussed by Marti et al. (1997) who covered a wide portion of the parameter space with $2 \mathrm{D}$ simulations. Threedimensional results for one of the cases studied by Marti et al. (1997) have been presented by Aloy et al. (1999). Their simulations generalize to the relativistic case typical results of the nonrelativistic regime, namely: (i) lighter jets exhibit fatter, nearly spherical cocoons (see e.g. Krause \& Camenzind 2003) as a consequence of the reduced head propagation velocity and bulk momentum of the beam; (ii) heavier jets advance at higher velocities generating cylindrical, thinner cocoons; (iii) high Mach numbers have the same effect as low density contrasts in producing elongated (spearhead) cocoons, while low Mach numbers jets form fatter cocoons (Massaglia et al. 1996). Marti et al. (1997) show that a similar behavior is found for relativistic jets, the main difference being that, increasing the Lorentz factor, jets, even at low density, tend to form cocoons that resemble those of the heavy non-relativistic case due to their increased inertia. In addition, in relativistic fluid dynamics, different Mach numbers correspond to different jet internal energies rather than beam velocities, this leaves the density contrast $\eta$ as the main parameter defining the shape of the cocoon.
Our results, as we shall see, confirm this global trend; however, in this scenario, we intend to focus our attention on the entrainment properties as a possible source of the jet deceleration. The entrainment process takes place from the interaction between the jet beam and the cocoon, promoted by the development of Kelvin-Helmholtz instabilities (KHI henceforth) at the beam interface. The entrained material is composed of jet backflowing material mixed with the shocked ambient medium through the contact discontinuity. The process is therefore quite complex and is determined by many different factors, the behavior of KHI of course being one of the most relevant. The linear analysis of the KHI for relativistic flows (Hardee 1987; Hardee et al. 1998; Hardee 2000) shows that the growth rate of unstable modes depends on the Mach number, density ratio between jet and external medium and Lorentz factor. In this respect, we intend to investigate for which set of parameters entrainment occurs more efficiently.

Obviously the numerical resolution is a very important factor in simulating the mixing processes. Therefore we perform our study with very high-resolution 3D computations in the hydrodynamic regime. The presence of a magnetic field may change the global structure, but as long as it is not too strong it is not believed to change the diffusion processes. Given the high computational cost of 3D simulations, we had to limit our study to a fixed value of the Lorentz factor varying only the Mach number and density ratio. Table 1 lists the complete set of parameters adopted in our numerical simulations, together with the mesh resolution. In the table, the first column gives the case identifier, the second column refers to Lorentz factor $\gamma_{b}$, the third to the Mach number, the fourth to the relativistic Mach number, the fifth to the ratio of proper densities, the sixth to number of mesh points on jet radius, the seventh the physical domain in units of jet radii and the last one the numerical domain. With the choice of these elements we can cover a complete set of combinations of Mach numbers and density ratios (cases A, B, $\mathrm{D}$ and $\mathrm{E})$. Moreover, case $\mathrm{C}$ represents a lower resolution version of case B, and a comparison between the two can provide indications of the role played by numerical resolution. In agreement with observations, we consider only jets with density that is much lower than the external medium; on the other hand high density jets propagate quasi ballistically and do not show instabilities and mixing.

As we discuss below, the main parameter governing the jet behavior and the entrainment properties turns out to be the density ratio $\eta$, while the Mach number plays a minor role even though it contributes to the determination of the KHI modes linear growth rates. Our discussion therefore refers to cases A and $\mathrm{B}$ as representatives of low and high $\eta$ values. Our results show that case $\mathrm{D}$ behaves very much like case $\mathrm{B}$, so we do not discuss it in detail. Case $\mathrm{E}$ is instead carefully discussed in order to show what the effects of a higher Mach number can be.

Figure 1 shows the volume rendering of the tracer distributions for cases A (top panel), B (mid panel) and E (bottom panel) when the evolutions have reached fully nonlinear stages. 

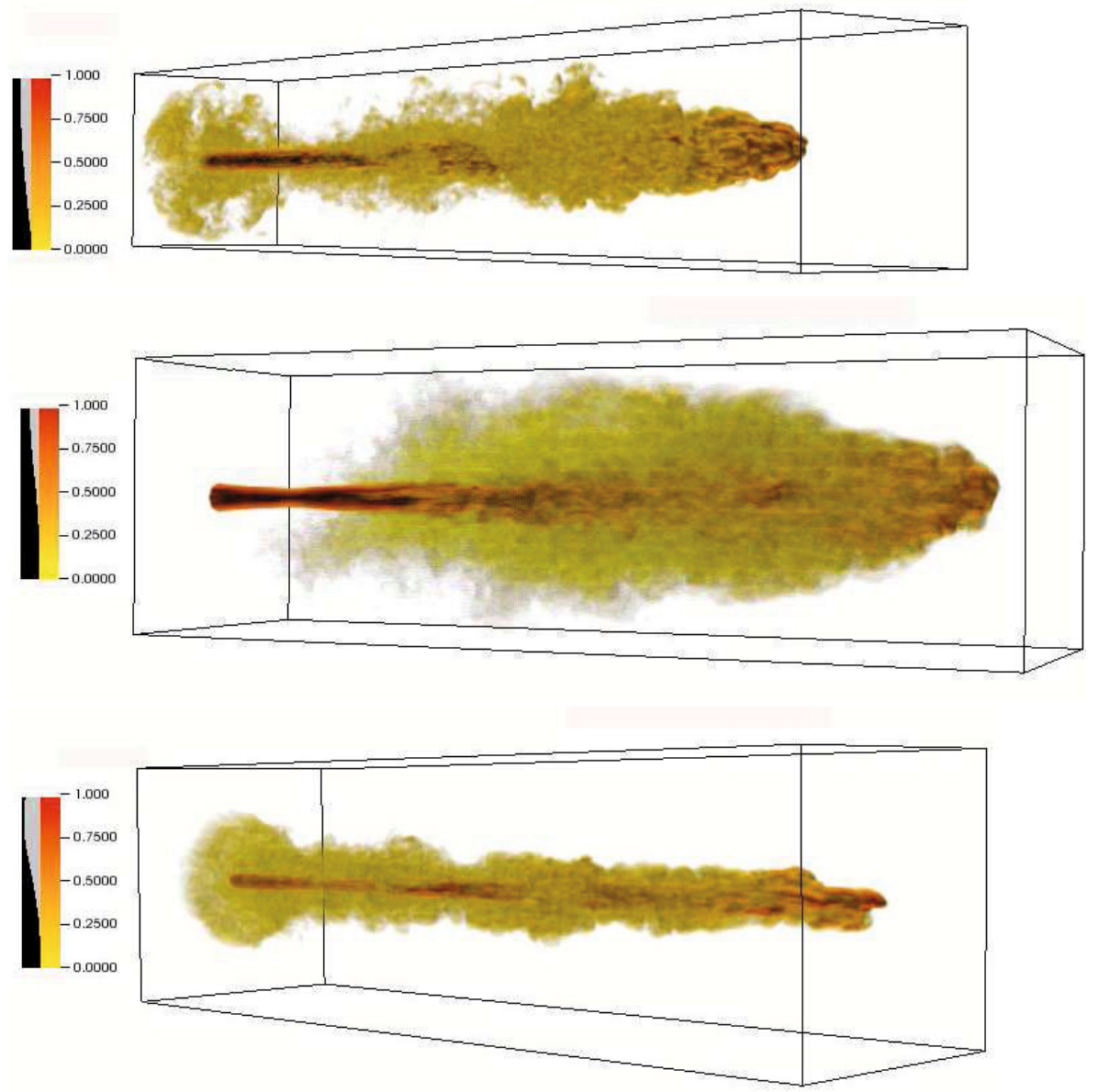

Fig. 1. Volume rendering of the tracer distribution for case A at $t=240$ (top panel), B at $t=760$ (central panel) and E at $t=150$ (bottom panel). Dark colors are due to the opacity of the material in the rendering procedure

Jet material is dark brown, external medium material white, and the level of mixing corresponds to the scale of orange. From these figures one can gain a first qualitative view about the role of the parameters on the evolution and structure of the jet: in cases $\mathrm{A}$ and $\mathrm{E}$ the jets propagate straight with moderate and low dispersion of jet particles, whereas in case B the jet has, at least partially, lost its collimation and the particle dispersion is considerably greater.

This is more clearly represented by the $2 \mathrm{D}$ cuts in the $x, y$ plane (at $z=0$ ) of the density and Lorentz factor distributions shown in Fig. 2. From the density (left) panels one can note that in both cases $\mathrm{A}$ and $\mathrm{E}$ the jet seems to be weakly affected by the perturbation growth and entrainment. This is not the situation for case B, where the beam structure is heavily modified by the growth of disturbances beyond $\sim 20$ jet radii. The distributions of the Lorentz factor (right panels) again indicate that the perturbation slightly affects the system in case A after $\sim 100$ radii, leaves the jet almost unchanged in case $\mathrm{E}$, and strongly influences the propagation after $\sim 20$ radii for case B, where the maximum value of $\gamma$ has reduced approximately to half of its initial value. However, even in case B a well collimated high velocity component along the axis still displays.

A quantitative estimate of the jet deceleration can be obtained by plotting the Lorentz factor as a function of the longitudinal coordinate $y$. Figure 3 shows the maximum value of $\gamma$ at constant $y$-planes together with its volume average, defined as

$\gamma_{\mathrm{av}}=\frac{\int \gamma g(\gamma) \mathrm{d} x \mathrm{~d} z}{\int g(\gamma) \mathrm{d} x \mathrm{~d} z}$

where $g(\gamma)$ is a filter function to select the relativistic flows:

$g(\gamma)= \begin{cases}1 & \text { for } \gamma \geq 2 \\ 0 & \text { for } \gamma<2 .\end{cases}$

For case B one can see that the deceleration occurs both in $\gamma_{\max }$ (the flow velocity, although still relativistic, shows a strong decrease from its initial value) and in $\gamma_{\mathrm{av}}$, which indicates a global effect, whereas in cases $A$ and $E$ the central part of the jet continues to be almost unperturbed and only thin external layers are decelerated.

As previously mentioned, the interaction between jet and surrounding medium involves different phenomena, reflecting the characteristics of the deceleration process: the growth rate of the perturbations, the type of perturbations that dominates the jet structure, the possibility of mixing through the backflowing material and the density of the ambient medium relative to the jet material. Long wavelength modes will tend to produce global jet deformations like jet wiggling more than mixing, while modes on a shorter scale may be more efficient in promoting the mixing process. On the other hand, it is clear that the mixing with a denser environment is more effective for the jet deceleration. 


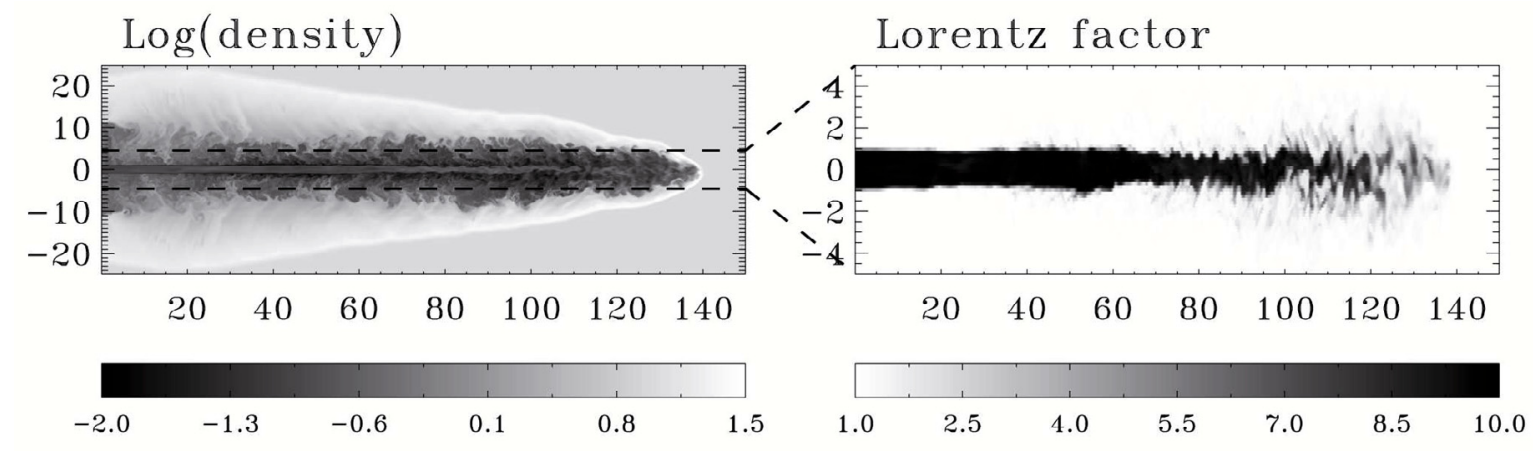

\section{$\log ($ density $)$}

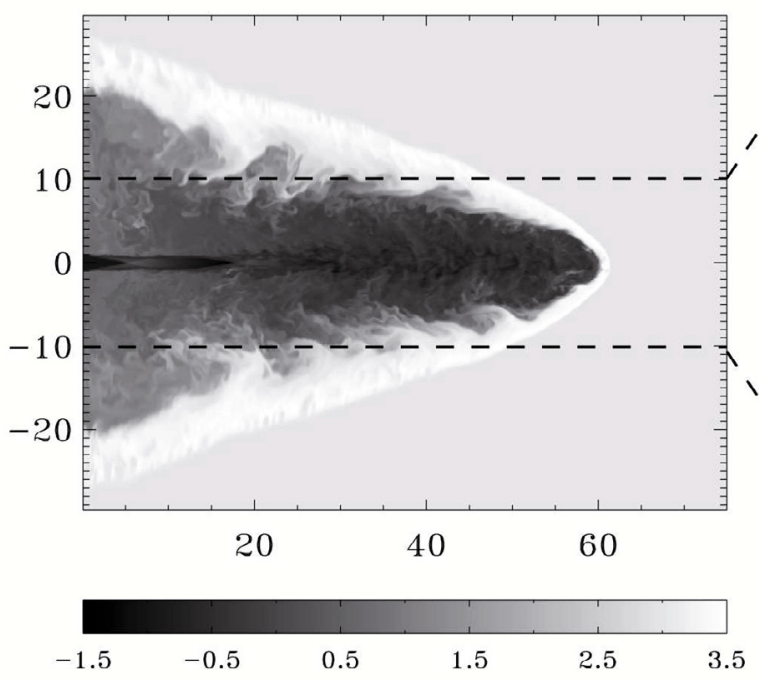

Lorentz factor

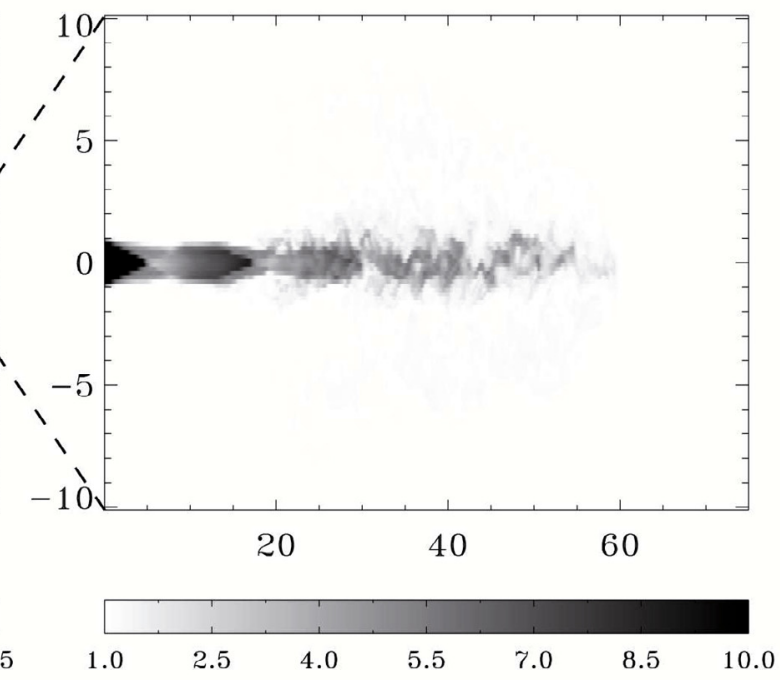

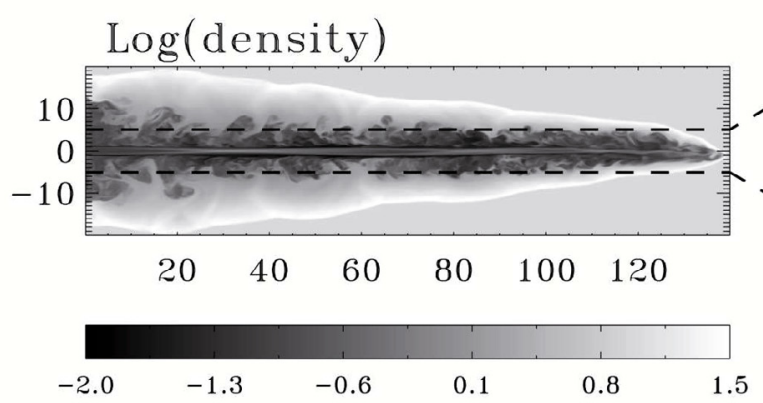

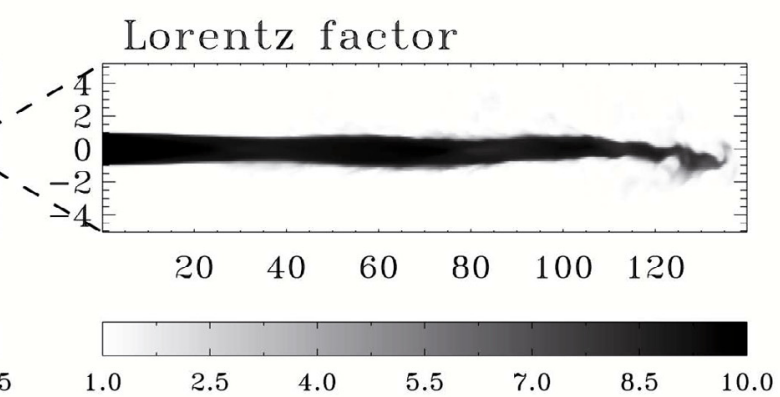

Fig. 2. Two-dimensional longitudinal cut in the $x y$ plane of the density distribution for case A at $t=240$ (top-left panel), case B at $t=760$ (centralleft panel) and case $\mathrm{E}$ at $t=150$ (bottom-left panel), and Lorentz $\gamma$ distribution in the central part of the domain, not in scale (the corresponding right panels).

One of the differences between the two cases that we are considering is, in fact, the type of modes that dominate the jet evolution: while case E exhibits longer wavelength and lower $m$ modes with a highly prominent helical distortion, in case B we observe shorter wavelength and higher $m$ modes. This behavior agrees with the linear analysis of Hardee (1987) that predicts that wavelengths of the resonant mode increases with the Mach number as $\lambda \propto \gamma M$. An impression of the characteristic scales of the dominant modes can be obtained from Fig. 4, where 3D contour images of the Lorentz factor are shown. The central panel, case B, shows that small scale structures dominate the structure throughout the jet, while case E (right panel) shows the dominance of the $m=2$ helical mode. The left panel (case A), denotes a less clear cut situation possibly due to the effect of the superposition of different modes, with the presence of structures with a scale smaller than in case $\mathrm{E}$.

So far we have compared our three simulations at a given time. In Figs. 5-10, we consider as well the temporal evolution of pressure, density and velocity, comparing the jet structures at three different times when they reach approximately equal lengths. In Fig. 5 we show cuts in the $(x, y)$ plane of the pressure distribution with superimposed contours of the Lorentz factor. The figure displays the formation of the typical series of regular biconical structures (Komissarov \& Falle 1998) produced by the overpressured cocoons squeezing the jet. Case B shows the highest pressure and has shocks of smaller obliquity. From the contour levels of the Lorentz factor we see once more that cases A and E keeps the initial value of 10 up to the jet head. Figure 6 

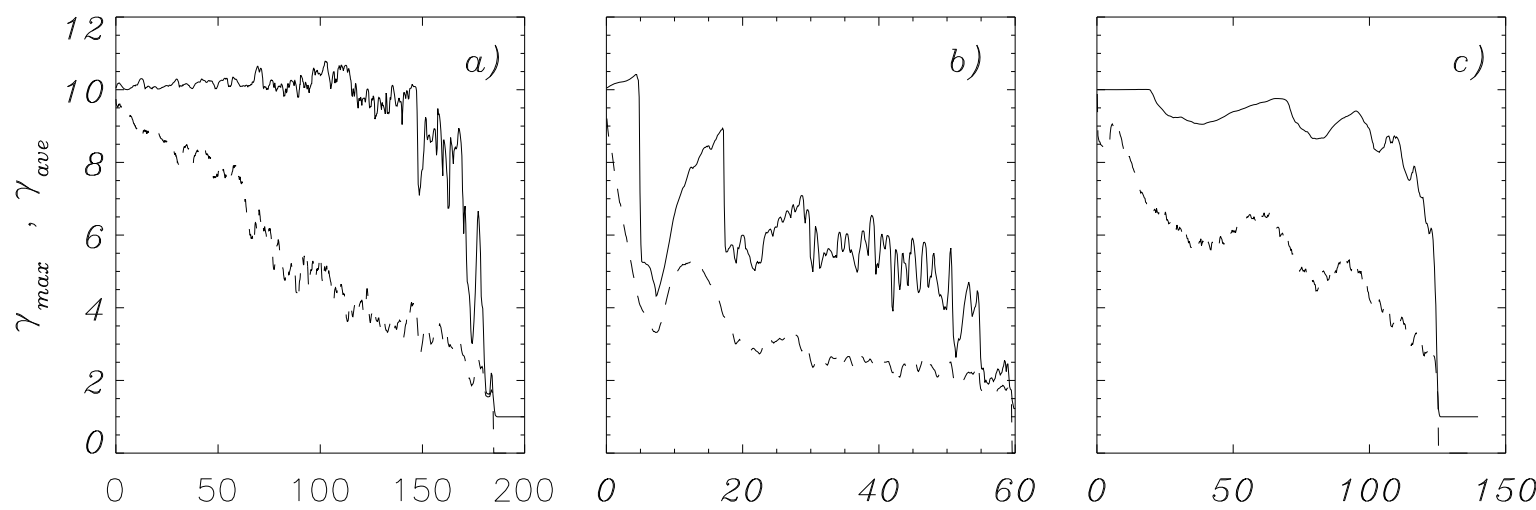

Fig. 3. Plots of the maximum value (solid line) and of an average value (dashed line) of $\gamma$ as functions of the longitudinal coordinate $y$ along the jet. The left panel refer to case A, the center panel to case B and the right panel to case E.
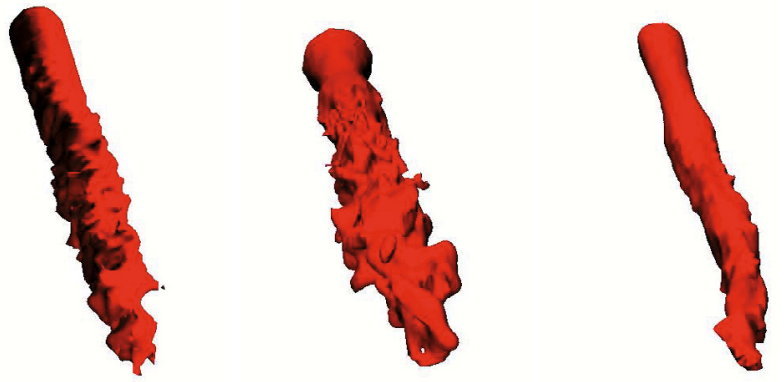

Fig. 4. Image of the Lorentz factor distribution of the contour for case A (left panel, $\gamma=5)$, case B (central panel, $\gamma=3$ ) and case $\mathrm{E}$ (right panel, $\gamma=5$ ).

shows the jets when they have reached a length of 120 radii; here we display only cases A and E, since the jet head velocity is very low for case $\mathrm{B}$, and the simulation was ended at a jet length of 60 radii. In the figure, we start seeing differences between case A, more perturbed, and case E, which shows almost no sign of interaction between the jet beam and the cocoon. Differences between cases $\mathrm{A}$ and $\mathrm{E}$ were already evident in Fig. 4, which shows the presence of small scale perturbations more favorable to mixing in case A and of only a long wavelength helical distortion in case E.

In contrast, for case B that shows the strongest deceleration, a first decrease in the Lorentz factor occurs at the first biconical shock (see also Figs. 2 and 3), the reacceleration following the shock, however, does not lead to a complete recovery of the initial value because the jet beam starts to interact with the cocoon and to lose momentum. We can note that, in the relativistic case, an increase in the jet pressure to resist squeezing by the overpressured cocoon would result in an increase in the jet energy flux (due to the relativistic contribution of pressure to inertia) and in a further increase in the cocoon pressure. Thus, in the relativistic regime, the formation of biconical shocks appears to be even more unavoidable than in the nonrelatistic regime.

The properties of the entrainment process can be better understood by looking at Figs. 7-10. In Figs. 7 and 8 we show cuts in the $(x, y)$ plane of the density distribution of the external medium (derived from the tracer distribution) for the three cases considered, at different times. This figure shows the ability of the external medium to penetrate the contact discontinuity and mix with the jet beam. We see that in B (central panel) the jet is surrounded by denser (green-red) external material in most of its length, while in cases A (left panels) and E (right panels) the medium around the jet is always lighter (blue-green). Comparing the different evolutionary stages (see Figs. 7 and 8), we also observe a slight decrease in density. The effect of the jet on the speed of entrained matter is shown in Figs. 9 and 10, where we display cuts of the distribution of the external medium velocity component along the jet axis. We note that the volume of the accelerated medium (red) is considerably greater in case B than in cases $\mathrm{A}$ and $\mathrm{E}$, consistently with a substantially stronger instability. Entrainment in case B already starts to be effective at a length of 30 radii, when it is almost absent in the other cases. At a length of 60 radii, apart from the dominant case B, we start observing differences between cases $\mathrm{A}$ and $\mathrm{E}$ with a stronger entrainment effect of the first. This difference becomes even more prominent at a length of 120 jet radii (see Fig. 10). From this figures we can also observe the formation of the backflow (blue region), more prominent in case $\mathrm{B}$ and almost absent in cases $\mathrm{A}$ and $\mathrm{E}$.

\section{Entrainment properties}

We now discuss the entrainment properties of the jet of case B $\left(M=3, \eta=10^{4}\right)$ in detail. We begin by examining the distribution in Lorentz factor and velocity of the jet and external material at $t=600$ (at the end of the simulation).

In Fig. 11 we show the distribution of the jet mass fraction that is moving at a certain value of $\gamma \beta$ at a given time. We use the four-velocity (instead of the Lorentz factor) to avoid compression of the scale close to $\gamma=1$, i.e. at low velocities. The color scale on the right gives the corresponding value of $\gamma \beta$ for each color. As an illustrative example, one can see that at $y=20$, $\sim 20 \%$ of the jet mass (per unit length) moves at $4<\gamma \beta<5$, $\sim 40 \%$ moves at $3<\gamma \beta<4, \sim 17 \%$ moves at $2<\gamma \beta<3, \sim 5 \%$ moves at $1<\gamma \beta<2$ and finally $\sim 18 \%$ moves at $0.1<\gamma \beta<1$. In this image one can clearly recognize the effect of reacceleration after internal shocks driven in the beam by the high pressure cocoon, particularly close to the inlet region. The central region of the jet never decelerate below $\gamma=4$, while in the external layers of the jet we observe the formation of an expanding region of low velocity material $(\gamma \beta<1)$.

Additional details are illustrated in Fig. 12, where the jet mass distribution is plotted as a function of $\gamma \beta$ at three different values of $y$ for two different instants.

At the first position $(y=12.5)$ the differences between the two times is relatively small, while at the two other locations we have a significant increase of the material moving at high $\gamma$. The jet in its first part has reached a quasi-steady configuration, while at larger distances from the source, after an initial deceleration phase, a well collimated high velocity core appears. Looking at 

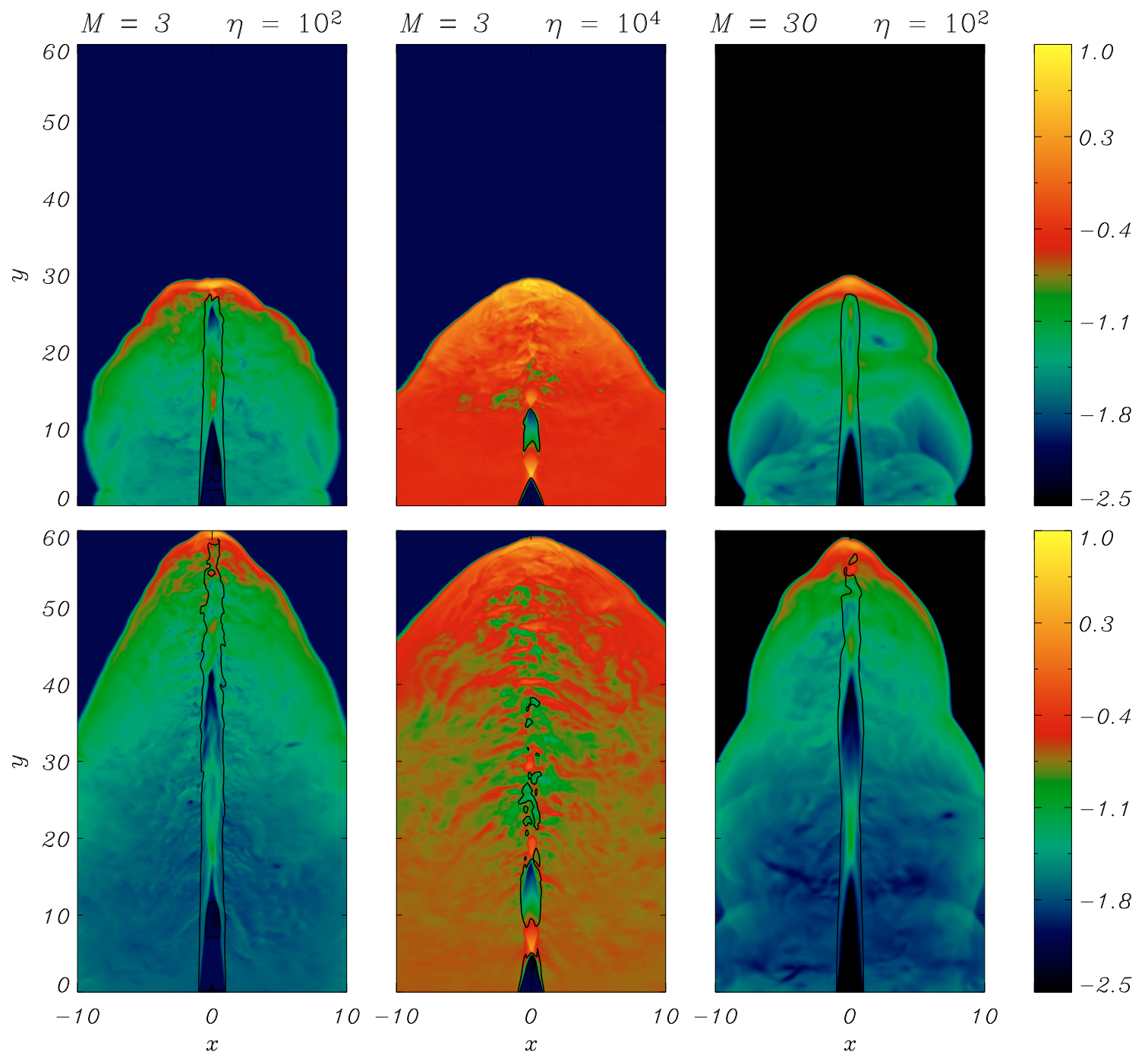

Fig. 5. Transverse cuts in the $x-y$ plane of the pressure distribution with superimposed the Lorentz factor contours. The left panels refer to case A, the middle panels to case $\mathrm{B}$ and the right panels to case $\mathrm{E}$. The upper panels show the jet when it has reached a length of $30 r_{j}$ while, in the lower panels, the jet length is $60 r_{j}$.
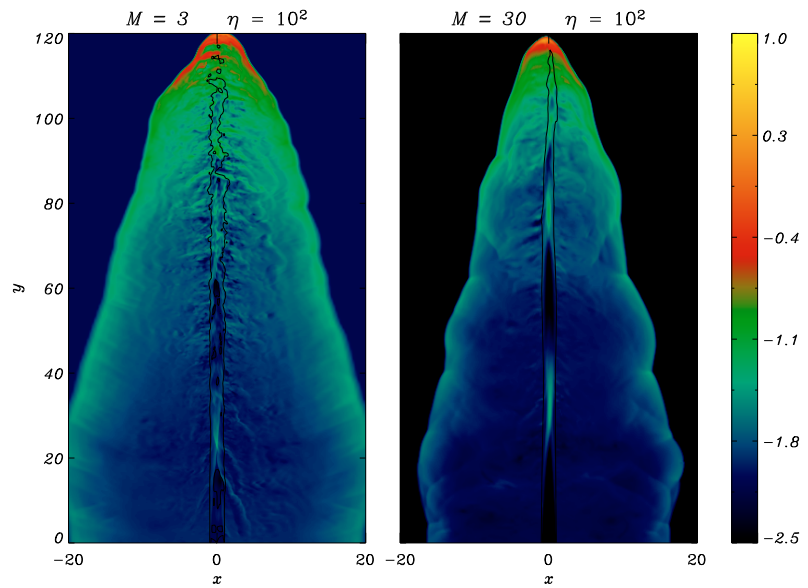

Fig. 6. Transverse cuts in the $x-y$ plane of the pressure distribution of the external material with the Lorentz factor contours superimposed. The left panel refers to case A, and the right panel to case E. The jet length is $120 r_{j}$.

the velocity structure, we see that, at the beginning ( $y=12.5$, see the left panel of Fig. 12), the jet is predominantly at high values of $\gamma \beta$, even though the deceleration mechanism starts to be effective, as shown by the low peak at $\gamma \beta \sim 0.2$. The effects of entrainment become much more evident at greater distances (see the two rightmost panels in Fig. 12), where we observe the formation of two sharp peaks, one at high $\gamma \beta$ and the other at velocities $\gamma \beta \sim 0.2$, with considerably less material at intermediate velocities. This implies that the jet structure has two well defined velocity components with a steep shear layer between them.

The distribution of external mass, analogous to Fig. 11 is also plotted, in Fig. 13. In this case all quantities are normalized to the jet mass injected in the domain per unit time.

The figure show that most of the external material is moving at quite low velocities, i.e. $0.1<\beta<0.3$. A similar analysis on the backflow reveals that most material moves with velocity in the range $-0.3<\beta<-0.1$, with a strongly dominant contribution given by the external medium. Higher velocity fluid elements with velocities up to -0.8 can be found in the front regions of the jet again with a dominant contribution by the ambient medium.

We conclude this section with a short evaluation of the effect of numerical resolution. Case $\mathrm{C}$ has the same parameters as case $\mathrm{B}$, but with only 12 points per jet radius, compared to 20 points per jet radius of case $\mathrm{B}$. Comparing the results obtained 

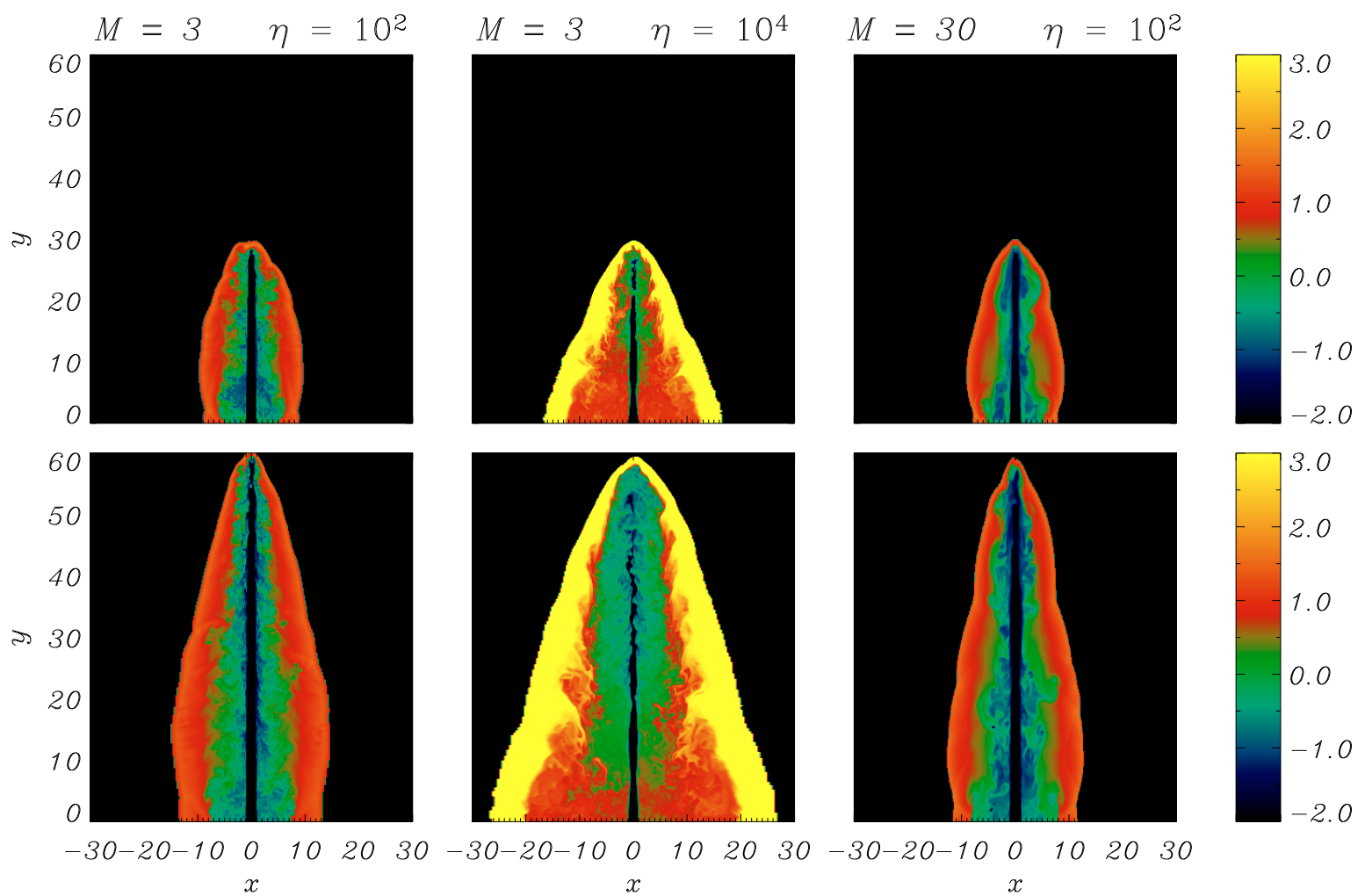

Fig. 7. Transverse cuts in the $x-y$ plane of the density distribution of the external material. The left panels refer to case A, the middle panels to case $\mathrm{B}$ and the right panels to case $\mathrm{E}$. The upper panels show the jet when it has reached a length of $30 r_{j}$ while, in the lower panels, the jet length is $60 r_{j}$.

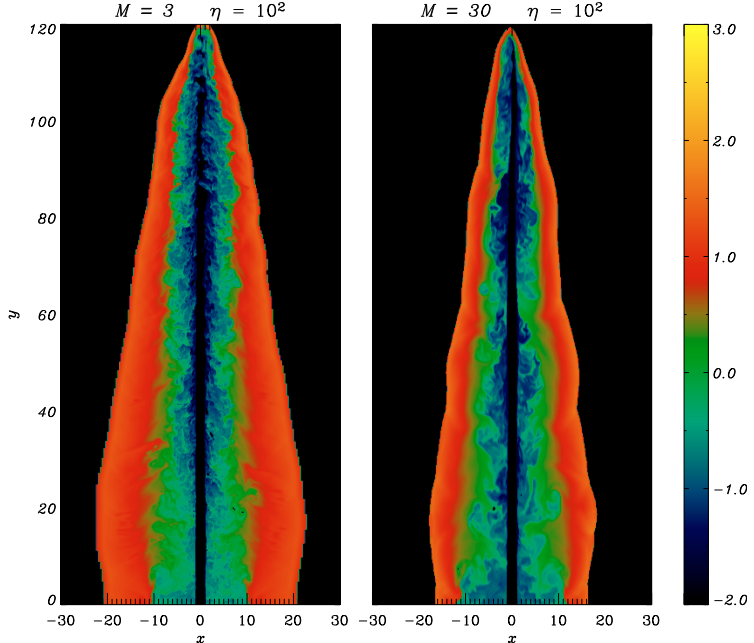

Fig. 8. Transverse cuts in the $x-y$ plane of the density distribution of the external material. The left panel refers to case $\mathrm{A}$, and the right panel to case $\mathrm{E}$. The jet length is $120 r_{j}$.

at these two different resolutions, we find that the general effect on the entrainment is to increase its efficiency when we increase the resolution. A detailed comparison shows that, for example, the average Lorentz factor decreases by about $15 \%$ in the higher resolution run. This effect can be interpreted observing that the most effective modes in term of entrainment are those at a shorter wavelength, which are under-resolved in a low resolution run.

\section{Astrophysical implications}

Recently several authors, e.g. (Chiaberge et al. 2000; Piner \& Edwards 2004; Giroletti et al. 2004), have proposed that observational properties of FR-I radio sources and their beamed counterparts (BL Lac objects) are produced by jets characterized by a velocity structure in which an inner core maintains a highly relativistic velocity and is surrounded by material that has been slowed down by the interaction with the ambient medium. A structure of this type is called the "spine-layer". The appearance of a jet with a spine-layer configuration is different when viewed at different angles. In fact, the two velocity components have different Doppler factors and the spine dominates the emission when the jet is observed at small angles with respect to the line of sight (BL Lac objects with strong Doppler boosting), while the prevailing contribution at larger angles is due to the entrained layer at low Lorentz factors (FR-I radio sources)

In our calculations, a "spine-layer" velocity structure has been obtained self-consistently as the result of a well defined physical process, i.e. the interaction of the outer jet layers with the ambient material, driven by jet instabilities. In particular we have found that, in the strongly underdense case $\eta=10^{4}$, the jet acquires a velocity structure in which the inner core maintains a highly relativistic velocity and is surrounded by material that has been slowed down by the interaction with the ambient medium. Therefore we attempt a comparison of radio maps constructed from the simulated jets with observations of FR-I jets. To this purpose, we compute synthetic maps by integrating the synchrotron emissivity along the line of sight. For the sake of simplicity we assume the emissivity to be proportional to the 


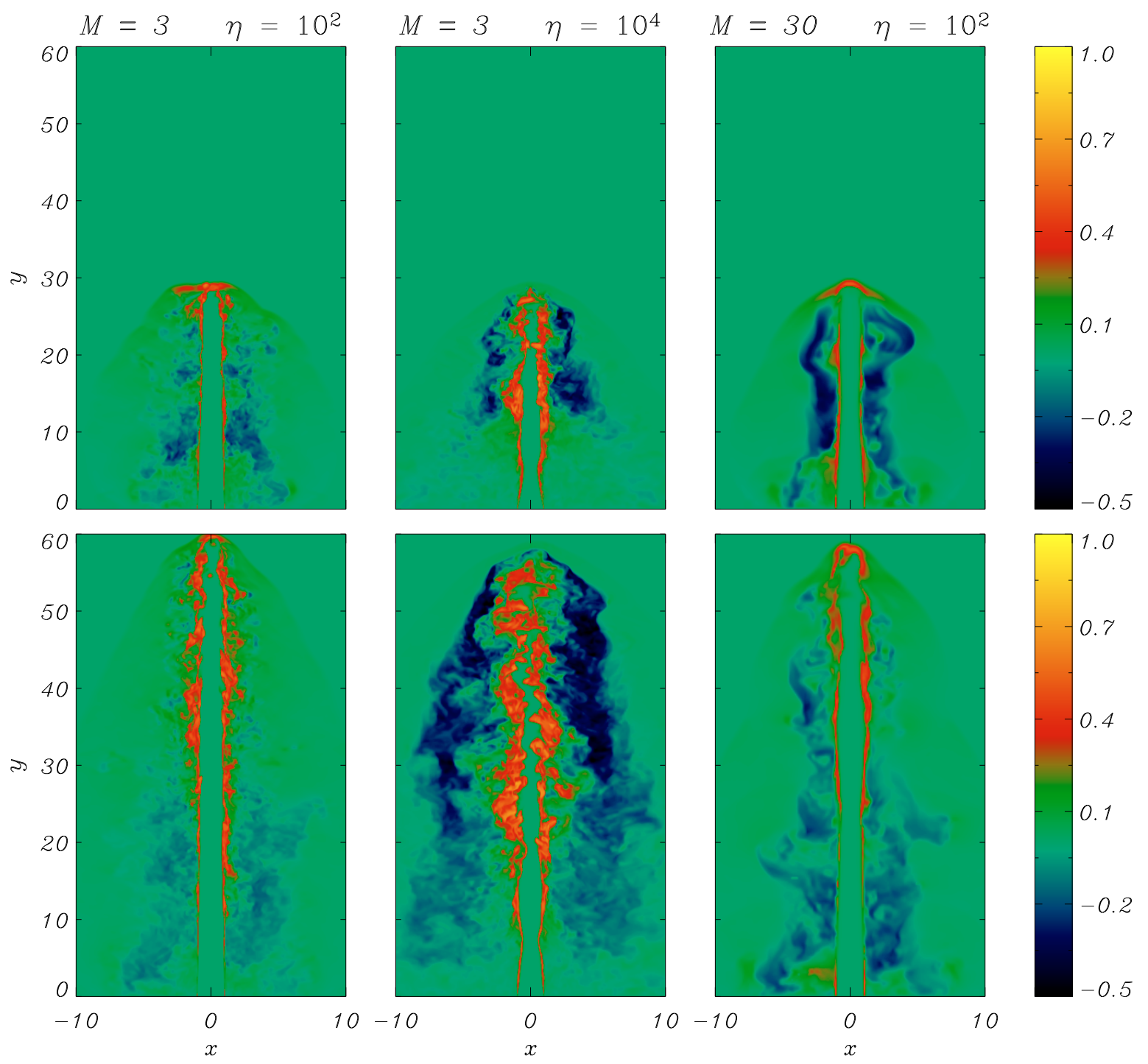

Fig. 9. Transverse cuts in the $x-y$ plane of the $v_{y}$ distribution of the external medium. The left panels refer to case A, the middle panels to case B and the right panels to case E. The upper panels show the jet when it has reached a length of $30 r_{j}$ while, in the lower panels, the jet length is $60 r_{j}$.

proper density of the jet material multiplied by the appropriate boosting or deboosting factor, i.e. by the quantity

$\epsilon(x, y, z)=\left[\frac{1}{\gamma(1-\beta \cos \theta)}\right]^{2+\alpha}$,

where $\alpha$ is the spectral index of the radio flux (we take $\alpha=0.5$ ). From this calculation we exclude the material at rest accumulated at the base of the jet as a result of the reflecting boundary condition imposed at $y=0$. The presence of this material is an artifact due to the imposed equatorial symmetry.

The synthetic maps for the strongly decelerated jets of case B are plotted in Figs. 14 and 15 for inclination angles of the jet axis to the line of sight of $20^{\circ}$ and $60^{\circ}$ respectively. The maps show a region with equal projected lengths of 45 jet radii, excluding the jet head, and therefore correspond to a longer jet in the small angle case. In the small angle case, the relativistic jet $\gamma \sim 4 \div 5$ core emission dominates. Emission is present along the whole jet and some knots can be recognized, the most brilliant being the first one, and knots correspond to the oblique shocks in the pressure maps. Increasing the inclination angle, the dominant contribution to emission is due to relatively slow material, whose fraction in the first part of the jet is very low, so that

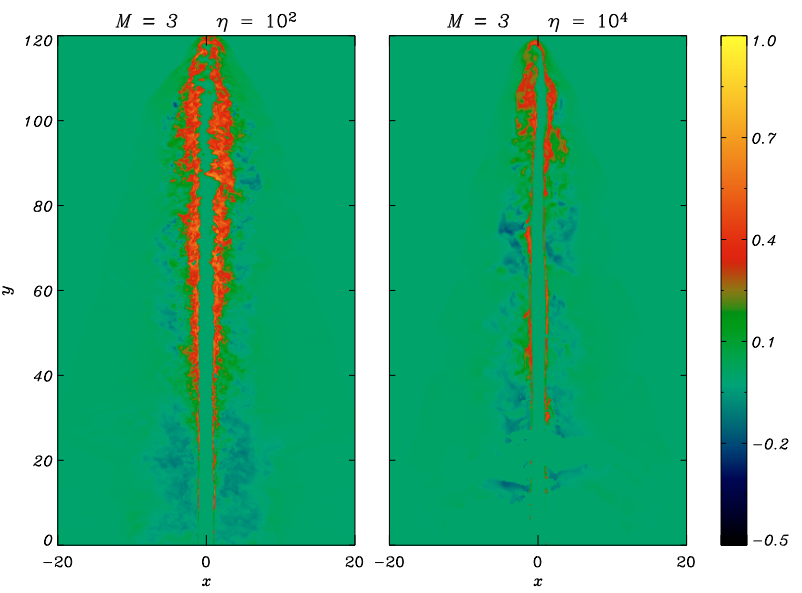

Fig. 10. Transverse cuts in the $x-y$ plane of the $v_{y}$ distribution of the external medium. The left panel refers to case $\mathrm{A}$, the and the right panel to case E. The jet length is $120 r_{j}$

emission is almost absent, apart from the first knot that is still visible at lower brightness. The fraction of slow material starts 

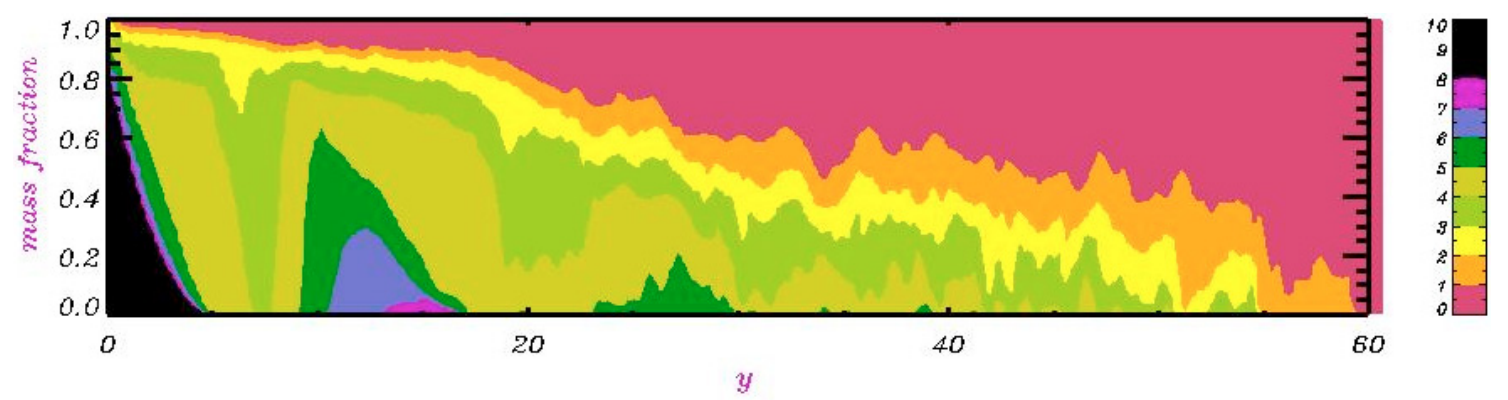

Fig. 11. Distribution of the jet mass fraction moving at a given $\gamma \beta$, when the jet length is $60 r_{j}$.
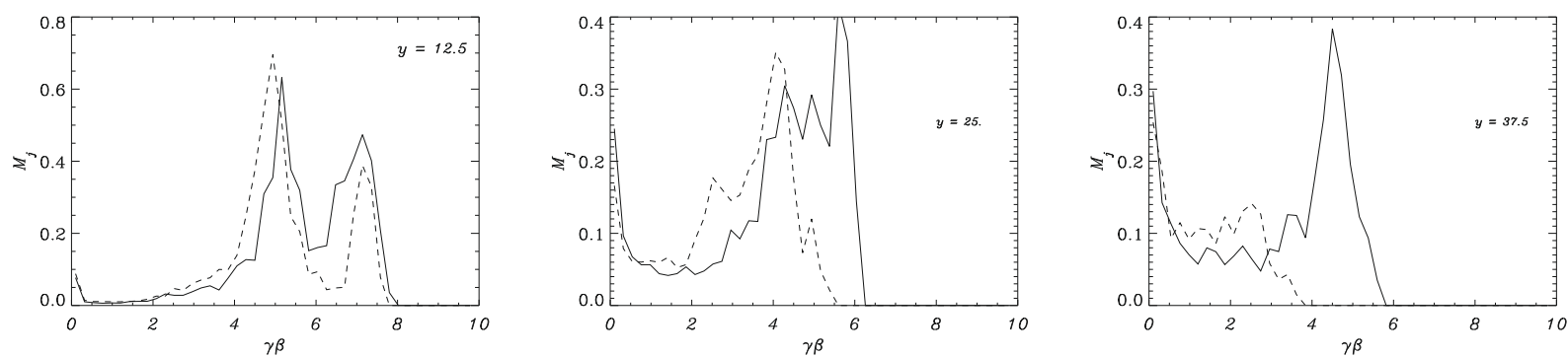

Fig. 12. Jet mass distribution as a function of $\gamma \beta$ at the locations $y=12.5,25,37.5$ at $t=400$ (dashed line) and $t=600$ (solid line).

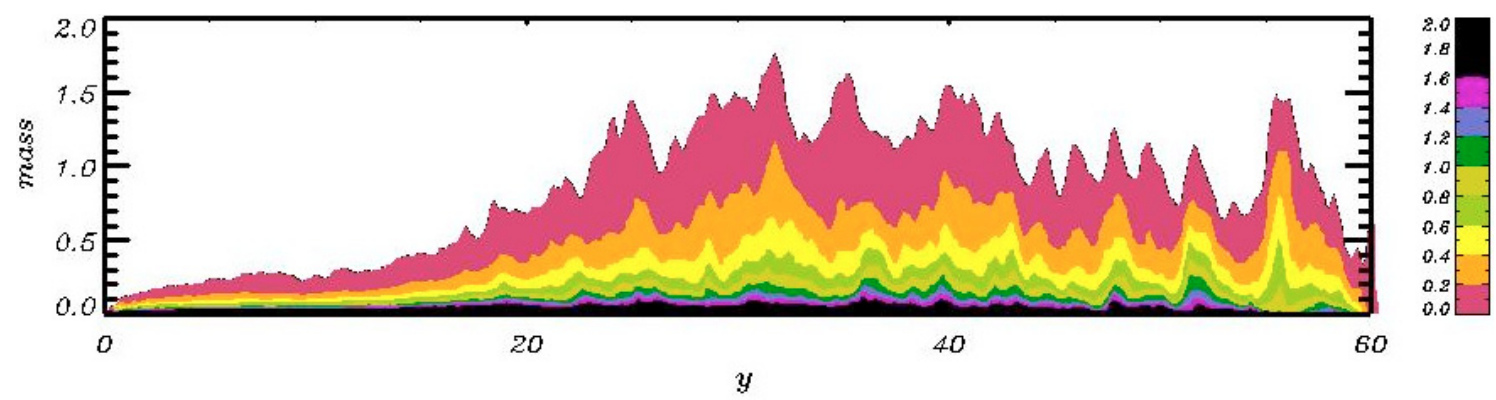

Fig. 13. The same as in Fig. 11 for the external medium and normalized with $M_{j}(0)$.

to increase at a distance of about 15 radii where the jet appears to have a sudden increase in opening angle, due to the formation of a thick layer of slowly moving material which dominates the emission at greater distances. We can also observe some limb brightening, explained by the emission coming from the slow layer surrounding the deboosted relativistic core.

We can compare these two synthetic maps with the radio maps of two typical FR-I sources shown in Figs. 16 and 17. The VLBA map of M87 can be compared with our synthetic map at a low inclination angle, which agrees with observational estimates. Instead the VLBI map of B2 $1144+35$ can be compared with our synthetic map at inclination of $60^{\circ}$, although the estimated inclination (Giovannini et al. 2007) for this source is $33^{\circ} \pm 7^{\circ}$. One of the possible origins of this discrepancy lies in the fact that the Lorentz factor of the slow layer in B2 1144+35 is estimated to be around 2.9 (the fast spine has $\gamma \sim 15$ ) corresponding to $\beta \sim 0.94$, while in our simulations (as shown in the previous section) the slow material is subrelativistic with $\beta \sim 0.3-0.4$ thus becoming visible at larger angles.

\section{Discussion and summary}

In this paper we have presented the $3 \mathrm{D}$ nonlinear dynamical evolution of relativistic light jets, as a result of a perturbation introduced at the jet inlet. The perturbation grows because of $\mathrm{KHI}$ and gives rise to a strong interaction of the jet with the

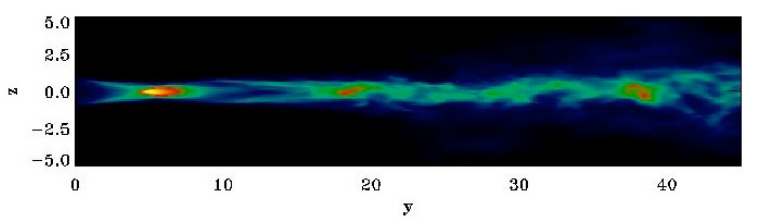

Fig. 14. Synthetic map for the jet of case B with an inclination of $20^{\circ}$ with respect to the line of sight.

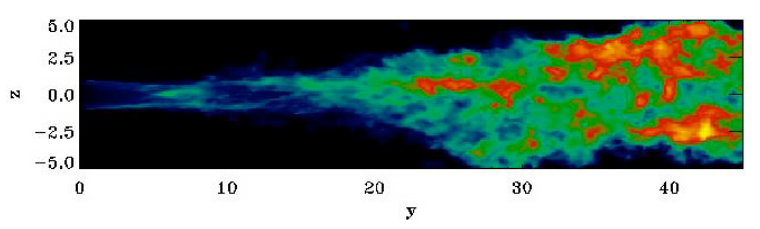

Fig. 15. Synthetic map for the jet of case B with an inclination of $60^{\circ}$ with respect to the line of sight.

external medium with a consequent mixing and deceleration. The two main parameters controlling the jet dynamics are the Mach number $M$ and the density ratio $\eta$ between the ambient medium and the jet. The Lorentz factor has been set equal to 10 in all computations.

We have explored the parameter plane $(M, \eta)$, finding that the deceleration becomes more efficient with increasing $\eta$. A 


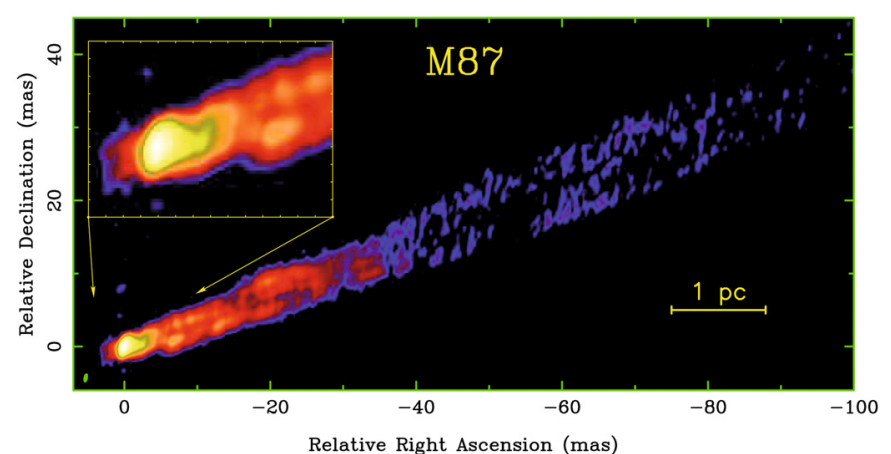

Fig. 16. VLBA radio image at $2 \mathrm{~cm}$ of M87 (Kovalev et al. 2007).

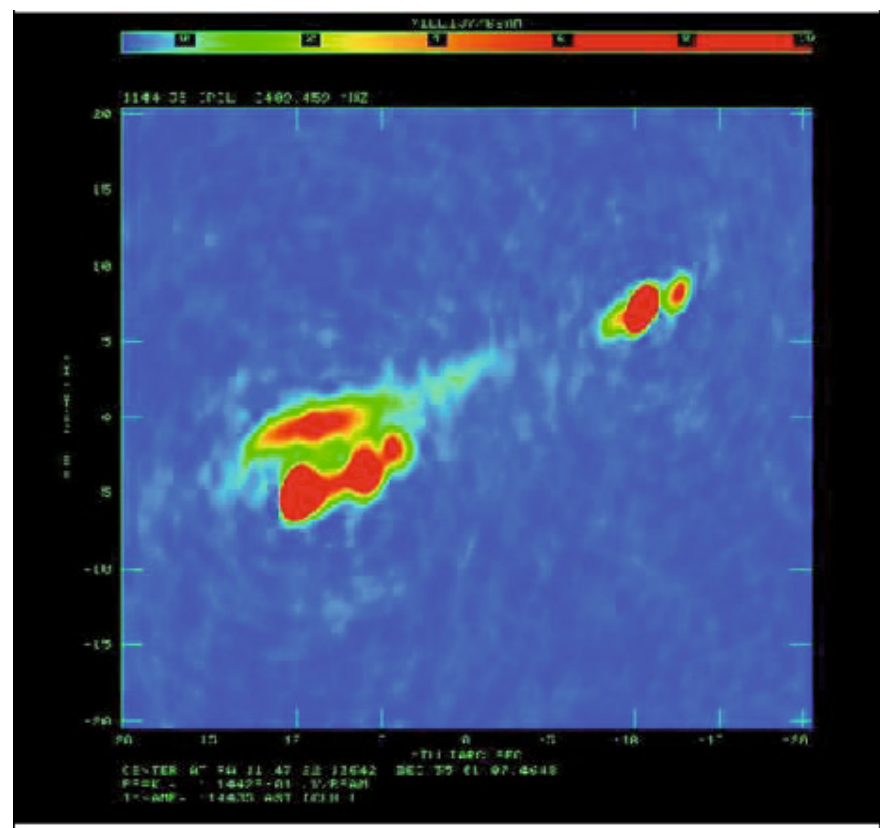

Fig. 17. VLBI image at $8.4 \mathrm{GHz}$ of B2 $1144+35$ (Giovannini et al. 2007).

preliminary analysis of the parameter space suggests that only jets with a high density ratio $\left(\eta>10^{2}\right)$ can undergo appreciable deceleration, while the Mach number does not seem to play a fundamental role in this respect.

We focused our attention on three extreme cases in the parameter plane, namely: case A with $M=3$ and $\eta=10^{2}$, B with $M=3$ and $\eta=10^{4}$ and $\mathrm{E}$ with $M=30$ and $\eta=10^{2}$. The comparison of these simulations in fact shows that case B (and D) undergoes the strongest deceleration. Cases $\mathrm{A}$ and $\mathrm{E}$ retain a high Lorentz factor spine with propagation velocities essentially unchanged from the injection value, while some deceleration has been observed only in the outer layers with the formation of a wide transverse velocity structure. In case B we observe the formation of a similar pattern, although at a much shorter distance and with a significant stronger decrease in the maximum Lorentz factor.

The fact that higher values of $\eta$ (i.e. lower jet densities) lead to prominent deceleration may have direct astrophysical implications. Observational data seem to indicate that the jet's kinetic power associated with FR-I radio sources is, on the average, $\sim 10^{3}$ times lower than in FR-II radio sources (Celotti 2003). On the other hand, there seems to be no difference in the value of the initial Lorentz factor in the two classes (Giovannini et al. 2001; Celotti \& Ghisellini 2008). Since lighter jet beams imply reduced jet kinetic powers, our model leaves the density contrast as the most likely candidate to account for the discrepancies in the deceleration process efficiency. Using some astrophysically relevant units we can rescale our models and come up with a rough estimate for the critical value of the jet power $P_{j}^{*}$ that separates FRI from FRII radiosources:

$P_{j}^{*} \sim 1.3 \times 10^{44}\left(\frac{r_{j}}{1 \mathrm{pc}}\right)^{2}\left(\frac{\gamma_{\mathrm{b}}}{10}\right)^{2}\left(\frac{n}{1 \mathrm{~cm}^{-3}}\right)\left(\frac{\eta^{*}}{10^{3}}\right)^{-1} \mathrm{erg} \mathrm{s}^{-1}$

where we assume a jet radius of $1 \mathrm{pc}$, an external density of $1 \mathrm{~cm}^{-3}$ that is typical at distances below $100 \mathrm{pc}$ (Balmaverde et al. 2008), and a critical density ratio separating the two behaviors of about $10^{3}$. The value of $P_{j}^{*}$ is affected by many uncertainities but agrees, for example, with the estimates given by Ghisellini \& Celotti (2001) based on the results by Willott et al. (1999), who gave for $P_{j}^{*}$ the value

$P_{j}^{*} \sim 10^{44}\left(\frac{M_{\mathrm{BH}}}{10^{8} M_{\odot}}\right) \operatorname{erg~s}^{-1}$

where $M_{\mathrm{BH}}$ is the mass of the central black hole.

Considering the case with $\eta=10^{4}$, we obtained a spine-layer structure similar to what is deduced from observations. Looking at the synthetic maps produced from the simulations, it is evident that many of the salient features are reproduced fairly well. The main difference lies in the terminal Lorentz factor of the slow layer, typically smaller than the observational estimates. In view of this first promising result we intend to pursue this investigation further, trying to constrain jet parameters better and introduce another essential ingredient: the magnetic field.

Acknowledgements. The numerical calculations were performed at CINECA in Bologna, Italy, thanks to INAF. This work has been partly supported by MIUR and the ASC FLASH Center at the University of Chicago.

\section{References}

Aloy, M. A., Ibanez, J. M. A., Marti, J. M. A., Gomez, J.-L., \& Mueller, E. 1999, ApJ, 523, L125

Balmaverde, B., Baldi, R., \& Capetti, A. 2008, A\&A

Bicknell, G. V. 1984, ApJ, 286, 68

Bicknell, G. V. 1986, ApJ, 300, 591

Bicknell, G. V. 1994, ApJ, 422, 542

Bicknell, G. V. 1995, ApJS, 101, 29

Biretta, J. A., Zhou, F., \& Owen, F. N. 1995, ApJ, 447, 582

Bodo, G., Massaglia, S., Ferrari, A., \& Trussoni, E. 1994, A\&A, 283, 655

Bodo, G., Massaglia, S., Rossi, P., et al. 1995, A\&A, 303, 281

Bodo, G., Rossi, P., Massaglia, S., et al. 1998, A\&A, 333, 1117

Bodo, G., Rossi, P., Mignone, A., Massaglia, S., \& Ferrari, A. 2003, New A Rev., 47, 557

Bowman, M., Leahy, J. P., \& Komissarov, S. S. 1996, MNRAS, 279, 899

Canvin, J. R., Laing, R. A., Bridle, A. H., \& Cotton, W. D. 2005, MNRAS, 363 , 1223

Celotti, A. 2003, New Astron. Rev., 47, 525

Celotti, A., \& Ghisellini, G. 2008, MNRAS, 385, 283

Chiaberge, M., Celotti, A., Capetti, A., \& Ghisellini, G. 2000, A\&A, 358, 104

De Young, D. S. 1996, in ASP Conf. Ser., 100 Energy Transport in Radio Galaxies and Quasars, 261

De Young, D. S. 2005, in X-Ray and Radio Connections ed. L. O. Sjouwerman K. K Dyer Published electronically by NRAO, http://www.aoc.nrao. edu/events/xraydio Held 3-6 February 2004 in Santa Fe, New Mexico, USA, (E7.01)

Fanaroff, B. L., \& Riley, J. M. 1974, MNRAS, 167, 31P

Ghisellini, G., \& Celotti, A. 2001, A\&A, 379, L1

Giovannini, G., Cotton, W. D., Feretti, L., Lara, L., \& Venturi, T. 2001, ApJ, 552, 508 
Giovannini, G., Giroletti, M., \& B., T. G. 2007, [arXiv:0708.3902v1]

Giroletti, M., Giovannini, G., Feretti, L., et al. 2004, ApJ, 600, 127

Hardcastle, M. J., Worrall, D. M., Kraft, R. P., et al. 2003, ApJ, 593, 169

Hardee, P. E. 1987, ApJ, 313, 607

Hardee, P. E. 2000, ApJ, 533, 176

Hardee, P. E., Rosen, A., Hughes, P. A., \& Duncan, G. C. 1998, ApJ, 500, 599

Harris, D. E., \& Krawczynski, H. 2006, ARA\&A, 44, 463

Komissarov, S. S. 1990, Ap\&SS, 171, 105

Komissarov, S. S. 1994, MNRAS, 269, 394

Komissarov, S. S. \& Falle, S. A. E. G. 1998, MNRAS, 297, 1087

Konigl, A. 1980, Physics of Fluids, 23, 1083

Kovalev, G., Lister, M. L., C., H. D., \& I., K. K. 2007, ApJ, 668, L27

Laing, R. A. 1993, in Sub-arcsecond Radio Astronomy, 346
Laing, R. A., \& Bridle, A. H. 2002, MNRAS, 336, 1161

Laing, R. A., Parma, P., de Ruiter, H. R., \& Fanti, R. 1999, MNRAS, 306, 513

Marti, J. M. A., Mueller, E., Font, J. A., Ibanez, J. M. A., \& Marquina, A. 1997, ApJ, 479, 151

Massaglia, S., Bodo, G., \& Ferrari, A. 1996, A\&A, 307, 997

Mignone, A. \& McKinney, J. C. 2007, MNRAS, 378, 1118

Mignone, A., Plewa, T., \& Bodo, G. 2005, ApJS, 160, 199

Mignone, A., Bodo, G., Massaglia, S., et al. 2007, ApJS, 170, 228

Piner, B. G. \& Edwards, P. G. 2004, ApJ, 600, 115

Rossi, P., Bodo, G., Massaglia, S., Ferrari, A., \& Mignone, A. 2004, Ap\&SS, 293, 199

Tavecchio, F., Maraschi, L., Sambruna, R. M., et al. 2004, ApJ, 614, 64

Urry, C. M. \& Padovani, P. 1995, PASP, 107, 803

Willott, C. J., Rawlings, S., Blundell, K., \& Lacy, M. 1999, MNRAS, 309, 1017 